EPJ Web of Conferences 113,05013 (2016)

DOI: $10.1051 /$ epjconf/201611305013

(C) Owned by the authors, published by EDP Sciences, 2016

\title{
Pseudoscalar mesons parton distribution amplitudes
}

\author{
Chen Chen ${ }^{1,2, a}$ and Chao $\mathrm{Shi}^{3,4, \mathrm{~b}}$ \\ ${ }^{1}$ Hefei National Laboratory for Physical Sciences at the Microscale, University of Science and Technology of \\ China, Hefei, Anhui 230026, People's Republic of China \\ ${ }^{2}$ Institute for Theoretical Physics and Department of Modern Physics, University of Science and Technology \\ of China, Hefei, Anhui 230026, People's Republic of China \\ ${ }^{3}$ Key Laboratory of Modern Acoustics, MOE, Institute of Acoustics, Nanjing University, Nanjing 210093, \\ China \\ ${ }^{4}$ Department of Physics, Nanjing University, Nanjing 210093, China
}

\begin{abstract}
We compute all kaon and pion parton distribution amplitudes (PDAs) to twistthree and find that all of the pion's distributions are symmetric and all of the kaon's distributions are skewed in favour of the heavier $s$-quark, which are clear signals of dynamical chiral symmetry breaking (DCSB). And we can also notice that only the pseudotensor PDA can reasonably be approximated by its conformal limit. At any realistic energy scale, the pseudovector and pseudoscalar PDAs differ markedly from the corresponding functions in QCD's conformal limit. We can use these PDAs as the inputs to study hard exclusive processes and get trustable results.
\end{abstract}

\section{Introduction}

The PDAs of pion and kaon are very crucial to study hard exclusive processes[1,2]. In the previous studies, people can only use the expressions of PDAs in the QCD's conformal limit[3, 4]. Now people can recalculate mesons' more realistic PDAs by using a new method. With this new method[5, 6], we calculate six PDAs of pion and kaon to twist-three.

\section{Distribution amplitudes and Bethe-Salpeter wave functions}

A pseudoscalar meson, $P_{\bar{g} f}(q)$, has three two-particle distribution amplitudes on the light front, they can be expressed as[7]

$$
\begin{aligned}
f_{P} \varphi_{P}(u) & =N_{c} \operatorname{tr} Z_{2} \int_{d k}^{\Lambda} \delta\left(n \cdot k_{\eta}-u n \cdot q\right) \gamma_{5} \gamma \cdot n \chi_{P}^{q}\left(k_{\eta}, k_{\bar{\eta}}\right) \\
i \rho_{P}^{\zeta} \omega_{P}(u) & =N_{c} \operatorname{tr} Z_{4} \int_{d k}^{\Lambda} \delta\left(n \cdot k_{\eta}-u n \cdot q\right) \gamma_{5} \chi_{P}^{q}\left(k_{\eta}, k_{\bar{\eta}}\right) \\
\frac{1}{4} \rho_{P}^{\zeta} v^{\prime}(u) & =N_{c} \operatorname{tr} Z_{4} \int_{d k}^{\Lambda} \delta\left(n \cdot k_{\eta}-u n \cdot q\right) \gamma_{5} \sigma_{\mu \nu} q_{\mu} n_{v} \chi_{P}^{q}\left(k_{\eta}, k_{\bar{\eta}}\right)
\end{aligned}
$$

a. e-mail: chenchen.anl@gmail.com

b. e-mail: shichao0820@gmail.com 


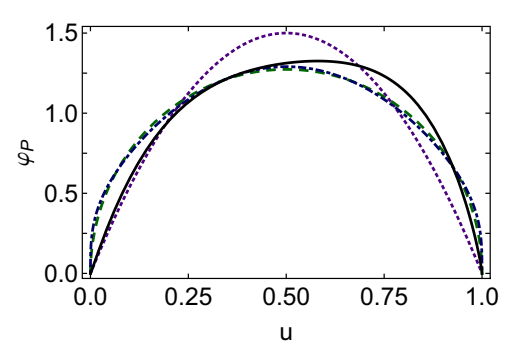

Figure 1. Two-particle twist-two PDAs, computed at $\zeta_{2}$ : dot-dashed curve (dark blue) - pion, $\varphi_{\pi}^{\mathrm{DB}}\left(u ; \zeta_{2}\right)$; solid curve (black) - kaon, $\varphi_{K}^{\mathrm{DB}}\left(u ; \zeta_{2}\right)$; dashed curve (dark green) $-\varphi_{\pi}^{\text {model }}(u)$, Eq. (5); and dotted curve (indigo) - asymptotic distribution, Eq. (4).

where $N_{c}=3$; the trace is over spinor indices; $\int_{d k}^{\Lambda}$ is a Poincaré-invariant regularisation of the fourdimensional integral, with $\Lambda$ the ultraviolet regularisation mass-scale; $Z_{2,4}(\zeta, \Lambda)$ are renormalisation constants; $\zeta$ is the renormalisation scale; $\chi_{P}^{q}\left(k_{\eta}, k_{\bar{\eta}}\right)$ is the Bethe-Salpeter wave function; and $f_{P}, \rho_{P}^{\zeta}$ are, respectively, the pseudovector and pseudoscalar projections of the meson's Bethe-Salpeter wave function onto the origin in configuration space. Every PDA should be normalized, viz.

$$
\int_{0}^{1} d u\left\{\varphi_{P}(u ; \zeta), \omega_{P}(u ; \zeta), v_{P}(u ; \zeta)\right\}=1
$$

We can numerically solve the coupled integral equations[8-11], namely, QCD's gap and BetheSalpeter equations, to get the expressions of quark propagators and the mesons' Bethe-Salpeter amplitudes. Here we solve these equations in two symmetry-preserving truncations[12]: the rainbow-ladder (RL) truncation, which is the most widely used in the calculation; and the modern DCSB-improved (DB) kernels, which can give the most realistic predictions by now.

Then we can employ the procedure in previous works to calculate PDAs[5, 6, 13]. First we need to calculate PDAs' moments

$$
\left\langle u_{\Delta}^{m}\right\rangle_{\phi}=\int_{0}^{1} d u(2 u-1)^{m} \phi_{P}(u),
$$

where $\phi=\varphi_{P}, \omega_{P}, v_{P}^{\prime}$, with these moments, we can reconstruct the PDAs by using Gegenbauer polynomials of order $\alpha,\left\{C_{n}^{\alpha}(2 u-1) \mid n=0, \ldots, \infty\right\}$. Gegenbauer polynomials form a complete orthonormal set on $u \in[0,1]$ with respect to the measure $[u(1-u)]^{\alpha_{-}}, \alpha_{-}=\alpha-1 / 2$. They therefore enable reconstruction of any function defined on $u \in[0,1]$.

\section{Numerical results}

\subsection{Pseudovector}

We depict our pseudovector results in Fig. 1, and compare them with the asymptotic two-particle distribution

$$
\varphi^{\mathrm{cl}}(u)=6 u(1-u)
$$

which is the asymptotic distribution in QCD's conformal limit, and with

$$
\varphi_{\pi}^{\text {model }}(u)=(8 / \pi) \sqrt{u(1-u)},
$$

which is a model result, it is practically indistinguishable from the DSE prediction. 

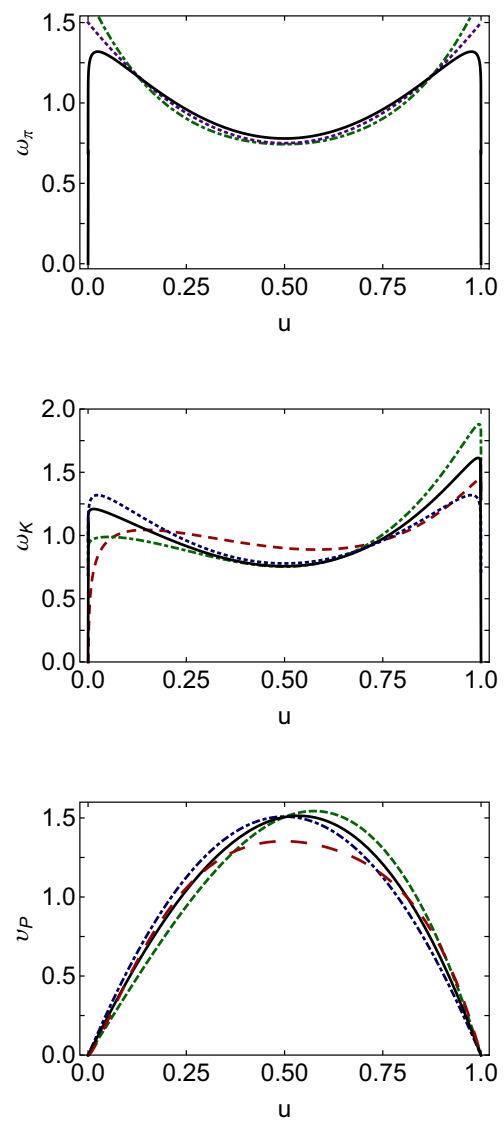

Figure 2. Pion two-particle, twist-three PDAs, computed at $\zeta_{2}$. Solid curve (black), $\omega_{\pi}\left(u ; \zeta_{2}\right)$; dot-dashed curve (dark green), QCD sum rules estimate [14]; and dotted curve (indigo), $\omega_{P}^{\text {asy }}$ in Eq. (6).

Figure 3. Kaon two-particle, twist-three PDAs, computed at $\zeta_{2}$. Solid curve (black), $\omega_{K}^{\mathrm{DB}}\left(u ; \zeta_{2}\right)$; dot-dashed curve (green), $\omega_{K}^{\mathrm{RL}}\left(u ; \zeta_{2}\right)$; dashed curve (red), QCD sum rules estimate [14]; and dotted curve (blue), $\omega_{\pi}\left(u ; \zeta_{2}\right)$.

Figure 4. Pseudotensor two-particle, twist-three PDAs, computed at $\zeta_{2}$ : dot-dashed curve (dark blue) - pion, $v_{\pi}(u)$; solid curve (black) kaon in DB truncation, $v_{K}^{\mathrm{DB}}(u)$; dashed curve (dark green) - kaon in RL truncation $v_{K}^{\mathrm{RL}}(u)$; and long-dashed curve (red) - QCD sum rules result for the kaon from Ref. [14]. We do not plot the asymptotic form because it is effectively indistinguishable from our prediction for the pion.

\subsection{Pseudoscalar}

We depict our pseudoscalar results in Fig. 2 and 3, and compare them with the asymptotic distribution

$$
\omega_{P}^{\text {asy }}(u)=1+\frac{1}{2} C_{2}^{(1 / 2)}(2 u-1)
$$

\subsection{Pseudotensor}

We depict our pseudotensor results in Fig. 4, we do not plot the asymptotic form because it is effectively indistinguishable from our prediction for the pion..

\section{Discussion and conclusion}

We can get many important information from our results. First, we can see that all of the pion's distributions are symmetric and all of the kaon's distributions are skewed in favour of the heavier $s$-quark, which are clear signals of DCSB. In kaon's pseudoscalar case, the RL PDA is more skewed than the DB result, viz. the RL truncation allocates more fraction of the in-kaon condensate to its valence $s$-quark. The RL-kernels ignore DCSB in the quark-gluon vertex, meanwhile the DB-kernels 
put DCSB into the quark-gluon vertex, so the the DB-PDA is more realistic. In kaon's pseudovector and pseudotensor case, we can get similar observations. It can be shown that in all PDAs, the $S U(3)$ flavour-symmetry breaking is a $13 \%$ effect $[5,15]$. And we can also notice that only the pseudotensor PDA can reasonably be approximated by its conformal limit. At any realistic energy scale, the pseudovector and pseudoscalar PDAs differ markedly from the corresponding functions in QCD's conformal limit.

Now we have calculated pion's and kaon's all the PDAs to twist-three, which are remarkably different from their expressions in the conformal limit except for the pseudotensor case. We can use these PDAs as the inputs to study hard exclusive processes and get trustable results.

\section{Acknowledgements}

The material described in this contribution is the result of an international collaborative effort that has involved, amongst others, L. Chang, G. Krein, S.-X. Qin, C. D. Roberts, J. Segovia, P.C. Tandy, S.-L. Wan and H.S. Zong. This work was supported by the Fundamental Research Funds for the Central Universities Programme of China (Grant No. WK2030040050); the National Natural Science Foundation of China (Grants No. 11275097 and No. 11475085); and the National Basic Research Programme of China (Grant No. 2012CB921504).

\section{References}

[1] G. P. Lepage and S. J. Brodsky, Phys. Lett. B 87, 359 (1979).

[2] A. V. Efremov and A. V. Radyushkin, Phys. Lett. B 94, 245 (1980).

[3] S. J. Brodsky, Y. Frishman, G. P. Lepage and C. T. Sachrajda, Phys. Lett. B 91, 239 (1980).

[4] V. Braun, G. Korchemsky and D. Mueller, Prog. Part. Nucl. Phys. 51, 311 (2003).

[5] L. Chang et al., Phys. Rev. Lett. 110, 132001 (2013).

[6] F. Gao, L. Chang, Y.-X. Liu, C. D. Roberts and S. M. Schmidt, Phys. Rev. D 90, 014011 (2014).

[7] V. M. Braun and I. Filyanov, Z. Phys. C 48, 239 (1990).

[8] P. Maris and C. D. Roberts, Int. J. Mod. Phys. E 12, 297 (2003).

[9] L. Chang, C. D. Roberts and P. C. Tandy, Chin. J. Phys. 49, 955 (2011).

[10] A. Bashir et al., Commun. Theor. Phys. 58, 79 (2012).

[11] I. C. Cloët and C. D. Roberts, Prog. Part. Nucl. Phys. 77, 1 (2014).

[12] L. Chang, C. D. Roberts and S. M. Schmidt, Phys. Rev. C 87, 015203 (2013).

[13] C. Shi et al., Phys. Lett. B 738, 512 (2014).

[14] P. Ball, V. M. Braun and A. Lenz, JHEP 0605, 004 (2006).

[15] C. Shi, C. Chen, L. Chang, C. D. Roberts, S. M. Schmidt and H.-S. Zong Phys. Rev. D 92, 014035 (2015). 